\title{
Inhibitory Effects of Far-Infrared Ray-Emitting Belts on Primary Dysmenorrhea
}

\author{
Ben-Yi Liau, ${ }^{1}$ Ting-Kai Leung, ${ }^{2}$ Ming-Chiu Ou, ${ }^{3}$ Cheng-Kun Ho, ${ }^{3}$ \\ Aiga Yang, ${ }^{3}$ and Yung-Sheng Lin $^{3}$ \\ ${ }^{1}$ Department of Biomedical Engineering, Hungkuang University, 34 Chung-Chie road, Shalu, Taichung 443, Taiwan \\ ${ }^{2}$ Department of Diagnostic Radiology, Taipei Medical University Hospital \& Department of Radiology, School of Medicine, \\ College of Medicine, Taipei Medical University, 250 Wu-Hsing Street, Taipei 110, Taiwan \\ ${ }^{3}$ Department of Applied Cosmetology and Master Program of Cosmetic Science, Hungkuang University, 34 Chung-Chie road, Shalu, \\ Taichung 443, Taiwan \\ Correspondence should be addressed to Yung-Sheng Lin, linys@sunrise.hk.edu.tw
}

Received 1 December 2011; Revised 21 May 2012; Accepted 5 June 2012

Academic Editor: Timon Cheng-Yi Liu

Copyright () 2012 Ben-Yi Liau et al. This is an open access article distributed under the Creative Commons Attribution License, which permits unrestricted use, distribution, and reproduction in any medium, provided the original work is properly cited.

This study investigated the therapeutic effect of the far-infrared ray-emitting belt (FIRB) in the management of primary dysmenorrhea in female patients. Forty adolescent females with primary dysmenorrhea were enrolled in the study. Quantitative measurements were taken during the menstruation. Several parameters were measured and compared, including temperature, abdominal blood flow, heart rate variability, and pain assessment. Statistical analysis shows that treatment with FIRB had significant efficiency in increasing regional surface temperature and abdominal blood flow, widening standard deviation of normal-to-normal RR intervals, and reducing VRS and NRS pain scores. The application of an FIRB appears to alleviate dysmenorrhea.

\section{Introduction}

Dysmenorrhea is one of the gynecological symptoms pertaining obviously to women. Ninety percent of women experienced menstrual pain, with a third to half of the women reporting moderate-to-severe symptoms. Population surveys suggest that, although prevalence rates vary considerably according to geographical locations, complaints of dysmenorrhea are widespread among diverse populations. Symptoms are frequently associated with time lost from school, work, or other activities. Despite the frequency and severity of dysmenorrhea, most women do not seek medical treatment for this condition.

"Dysmenorrhea" is derived from a Greek root word that translates as "difficult menstrual flow." Dysmenorrhea can be divided into the two broad categories of primary and secondary. Primary dysmenorrhea is defined as a cramping pain in the lower abdomen occurring just before or during menstruation, without pelvic abnormalities. Secondary dysmenorrhea is menstrual pain associated with underlying pelvic pathology such as endometriosis.
Primary dysmenorrhea is typically characterized by a menstrual cramp that begins between several hours before and a few hours after onset of menstrual bleeding. Primary dysmenorrhea may be associated with vomiting, fatigue, back pain, headaches, dizziness, and diarrhea. Ninety percent of young women report that the duration of their menstrual cramps is 48 hours or less. Symptoms are relatively repeated from one menstrual period to the next. The pain is characteristically colicky and located in the midline of the lower abdomen. Primary dysmenorrhea arises from the secretion of prostaglandins during the luteal phase and subsequent menstrual flow. Excessive release of prostaglandins increases the amplitude and frequency of uterine contractions and causes vasospasm of the uterine arterioles, resulting in ischaemia and cyclical lower abdominal cramps.

In the United States, dysmenorrhea accounts for 600 billion working hours loss and over US\$ 2 billion productivity lost every year [1]. In Taiwan, primary dysmenorrhea is the most common reason adolescent girls consult gynecologists. Dysmenorrhea may lead to severe anxiety; 
thus, if pain during menstruation can be relieved, anxiety levels may also be reduced [2]. Many women consider menstrual pain to be inevitable even though the pain is severe and incapacitating. Women suffering from primary dysmenorrhea may not seek medical assistance and frequently do not use available prescription therapies. Antiinflammatory drugs are generally the first choice of treatment for primary dysmenorrhea. Anti-inflammatory drugs directly inhibit the enzyme of cyclooxygenase (COX-2), indirectly reducing prostaglandin synthesis. However, 20\% to $30 \%$ of patients with primary dysmenorrhea report that anti-inflammatory drugs are ineffective. In addition, drug therapy provides only temporary relief, and drugs are associated with several gastrointestinal side effects and complications such as anti-inflammatory-induced gastric ulcers or bleeding, and increased load on the kidneys and liver. Thus, an effective alternative therapy without drugs is necessary for the treatment of dysmenorrhea.

Research has shown that electromagnetic waves of farinfrared ray (FIR) especially at $4-14 \mu \mathrm{m}$ produce both nonthermal and thermal effects [3-9]. Such effects include an increase in microvascular dilation, higher blood flow volume, and an elevation in regional tissue temperature [35]. The consensus view is that these wavelengths promote other intracellular effects at the microscopic level, affecting heat transfer in subcutaneous tissues, and other physiobiological processes $[5,6,10]$. Several studies indicated that farinfrared ray irradiation is an effective treatment for chronic pain [10-13]. However, a limited number of studies have used objective parameters to assess the clinical efficacy of FIR, or have attempted to explain its mechanism on a biomolecular basis.

This study investigated the therapeutic effect of an FIR-emitting belt (FIRB) on dysmenorrhea by analyzing objective parameters and attempted to explain the possible mechanisms. In this study, participants were assessed during the dysmenorrheal period, and changes were monitored before and after application of the FIRB. Dysmenorrhea was measured quantitatively by thermography, laser Doppler imaging, standard deviation of normal-to-normal RR intervals (SDNN), verbal rating scale (VRS), and numeric rating scale (NRS).

\section{Materials and Methods}

2.1. Participants. The clinical trial was approved by an independent ethics committee of the university, with certification by the Institutional Review Board (IRB) approval number HK IRB 98-B-002. We performed this experiment from February 1, 2010 to January 31, 2011.

Participants were recruited for the study cohort from campus. Adolescent female students with dysmenorrhea volunteered as candidates and were screened by the doctor. All of the participants were required to complete a pretest questionnaire on their medical history and pain. The questionnaire investigated possible discomfort and other descriptions. The participants were also required to sign a consent form before any measurements were taken or FIRB

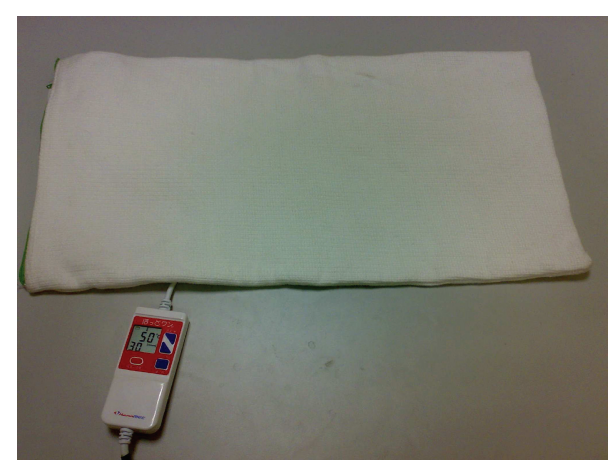

FIGURE 1: The photograph of the carbon fiber belt in this study. The belt controller was set to $50^{\circ} \mathrm{C}$ for 30 minutes.

was applied. The inclusion criteria for all candidates who participated in this study were as follows: older than 18; selfreported dysmenorrhea; no history of major gynecological disease or secondary dysmenorrhea; no pain medication taken in the 24 hours before receiving treatment. Only subjects on the first-to-third days from the onset of menses could be included in this study to treat with the FIRB. The carefully selected sample included forty young women with primary dysmenorrhea.

2.2. FIR-Emitting Belt. The FIR spectrum of the carbon fiber fabric belt $(30 \mathrm{~cm} \times 60 \mathrm{~cm}$ as shown in Figure 1, THERMEDIC, LinkWin Technology Co., Ltd., Taiwan) used in this study was determined by a CI SR5000 spectroradiometer at the Industrial Technology Research Institute, Taiwan. The FIR energy generated from the carbon fiber belt at $50^{\circ} \mathrm{C}$ is $11.34 \mathrm{~mW} / \mathrm{cm}^{2}$ by integrating the intensity of the wavelengths between $4 \mu \mathrm{m}$ and $16 \mu \mathrm{m}$ in Figure 2 .

2.3. Measurements. All measurements and procedures in this study were performed in a climate-controlled room at a constant temperature and humidity $\left(23^{\circ} \mathrm{C}\right.$ and $60 \%$ relative humidity). Before assessment, participants were required to lie down in this room to acclimatize to these indoor climatic conditions for 30 minutes [14]. Participants were thermographed for the abdominal region by the Fluke Ti25 (Fluke Corporation, Everett, WA, USA). Their abdominal blood flows were measured by the MoorLDI2-IR laser Doppler imaging (Moor Instruments Ltd., Devon, UK) [15]. Subsequently, they were asked to wear the FIRB on the abdominal region. Then FIRB was warmed to a temperature of $50^{\circ} \mathrm{C}$ to enhance FIR irradiation for 30 minutes. The second measurements of abdominal temperature and blood flow were performed after the application of FIRB.

In addition, ANSWatch wrist monitor (TS-0411, Taiwan Scientific Corp., Taiwan) utilized in studying nervous system regulation $[16,17]$ was applied to conduct a standard 5minute heart rate variability (HRV) test with left hand before and after using FIRB for 30 minutes. The cuff with piezoelectrical sensors obtained blood pressure signals produced by the radial artery, with the aid of an air pouch pressure controlled by an air pump and a release valve. The blood pressure waveforms with peak-to-peak intervals were determined in 


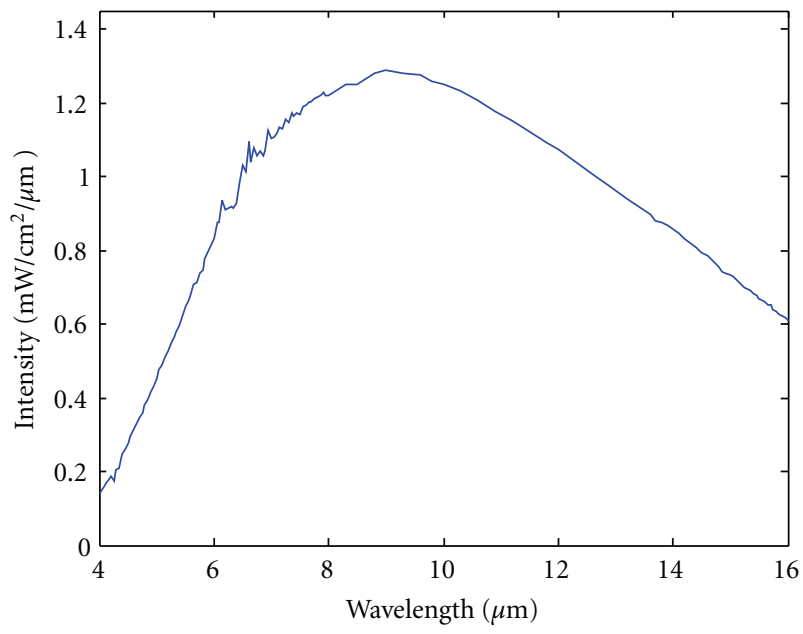

Figure 2: The FIR spectrum of the carbon fiber belt at $50^{\circ} \mathrm{C}$. The total intensity of the wavelengths between $4 \mu \mathrm{m}$ and $16 \mu \mathrm{m}$ is $11.34 \mathrm{~mW} / \mathrm{cm}^{2}$

time domain analysis. The HRV analysis followed closely the 1996 International Standard. HRV is total ANS activity index (ms) that equal to standard deviation of normal-to-normal RR intervals defined in the 1996 international standard [18].

2.4. Menstrual Pain Assessment. Before and after the application of FIRB, all the participants were required to complete two pain questionnaires to assess the pain scores associated with their dysmenorrhea. These pain assessments were the five-level verbal rating scale (VRS) and 11-point numeric rating scale (NRS) [19]. The VRS pain scale was classified into grades of none (0), very mild (1), mild (2), moderate (3), severe (4), and very severe (5). The NRS was divided into eleven levels of pain, ranging from no pain (0) to the worst pain (10).

2.5. Statistical Analysis. The data differences between before and after treatment were analyzed using Wilcoxon signedrank test. A value of $P<0.05$ was considered statistically significant $(*)$, and $P<0.01$ was highly significant $(* *)$.

\section{Results}

3.1. Effect of FIRB on Abdominal Temperature. The abdominal temperature was measured by thermography (Fluke Ti25). Results indicated that there was a substantial increase in regional body temperature after participants used FIRB for 30 minutes. It was shown in Figure 3. The average abdominal temperatures of before and after FIRB treatment were $34.6 \pm$ $1.2^{\circ} \mathrm{C}$ and $37.5 \pm 1.2^{\circ} \mathrm{C}$, respectively. This temperature increase was safe for skin and did not cause any burns in participants. There was no side effect such as menorrhagia reported in the treatment of primary dysmenorrhea with the FIRB.

3.2. Effect of FIRB on Blood Flow. The abdominal blood flow was measured by the Moor laser Doppler imaging device (MoorLDI2-IR). A significant increase was found in

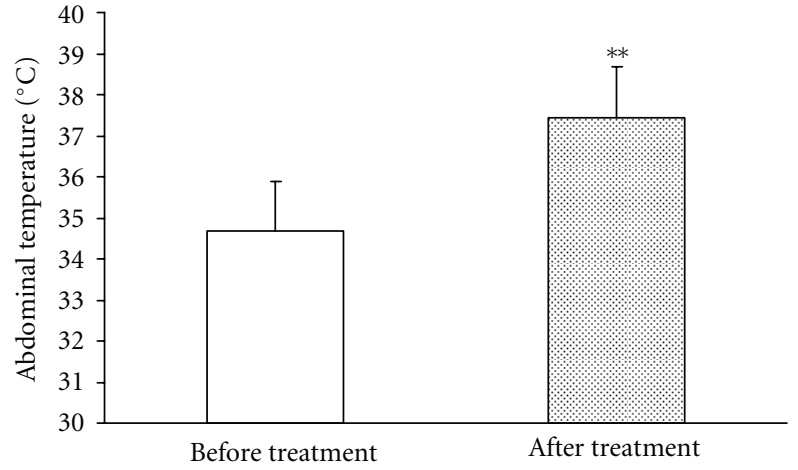

Figure 3: The average abdominal temperatures before and after treatment. Expression values are the mean and standard deviation, and the statistical difference was analyzed by Wilcoxon signed-rank test. Double asterisk mean that this group was highly significantly different from control group $(P<0.01)$.

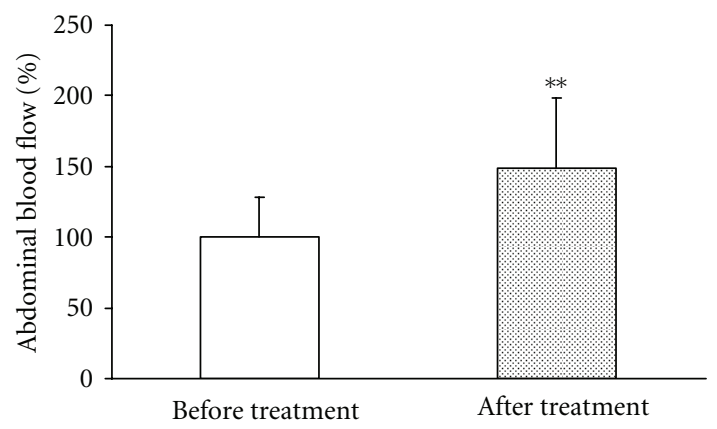

Figure 4: The relative abdominal blood flows before and after treatment. Expression values are the mean and standard deviation, and the difference between groups was tested using Wilcoxon Signed-Rank Test. Double asterisk mean that this group was highly significantly different from control group $(P<0.01)$.

regional blood perfusion after participants' use of FIRB for 30 minutes (Figure 4 ). There was a $48.7 \pm 49.7 \%$ increase in the normalized abdominal blood flow after FIRB treatment.

3.3. Effect of FIRB on Physiological Signals. No significant differences emerged in the physiological parameters of the blood pressure and respiration between before and after FIRB treatment conditions. However, the standard deviation of normal-to-normal RR intervals in pulse waveforms increased significantly (Figure 5). The average SDNN values of before and after FIRB treatment were $69.2 \pm 42.2 \mathrm{~ms}$ and $97.2 \pm 72.8 \mathrm{~ms}$, respectively. This finding may imply that the autonomic nervous system became more active with treatment. It seems possible that FIRB may help relieve pain by regulating the autonomic nervous system.

3.4. Effect of FIRB on VRS and NRS Scores. The pain assessments by VRS and NRS showed that treatment with FIRB significantly decreased the participants' subjective ratings of their pain or suffering associated with dysmenorrhea (Figures 6 and 7). The average VRS scores before and after FIRB treatment were $1.89 \pm 1.02$ and $0.76 \pm 0.68$, 


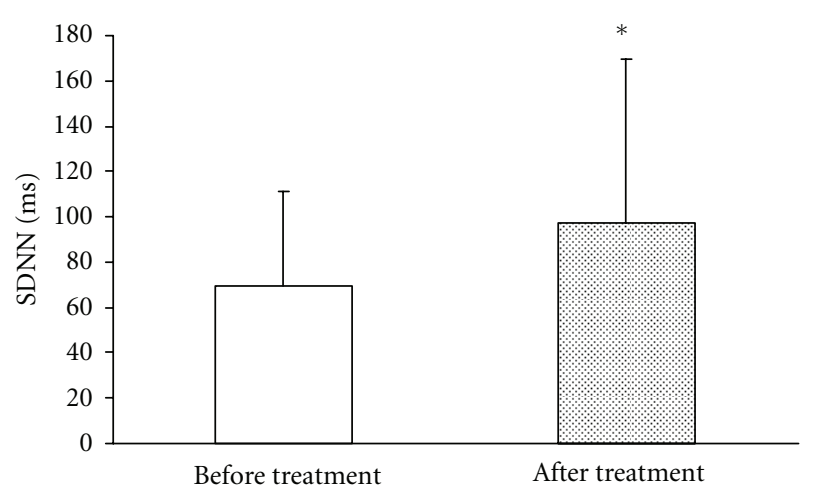

FIgURE 5: The mean SDNN values before and after treatment. Expression values are the mean and standard deviation, and Wilcoxon Signed-Rank Test was used to test the statistical difference between groups. Asterisk means that this group has statistical difference with control group $(P<0.05)$.

respectively. The average NRS scores before and after FIRB treatment were $4.57 \pm 1.83$ and $1.62 \pm 1.62$, respectively.

\section{Discussion}

The etiology of primary dysmenorrhea is bounded in a multiplicity of pathological, mechanical, and psychoneurotic disorders. One disorder that has been found to correlate with primary dysmenorrhea is blood flow deprivation with organic and lower abdominal ischemia. The sensation of pain and related biomolecule induction of COX-2 and prostaglandin are strongly related to the severity of primary dysmenorrhea. Previous studies have identified the overproduction of uterine prostaglandins as a contributing factor to primary dysmenorrhea. The most widely used anti-inflammatory drugs for primary dysmenorrhea directly inhibit the cyclooxygenase (COX) enzymes, thereby inhibiting the production of prostaglandins. Prostaglandin synthesis is mediated primarily by two distinct isoforms of cyclooxygenase (COX-1 and COX-2), which catalyze the metabolism of arachidonate to prostaglandin $\mathrm{H} 2$. Conventional anti-inflammatory drugs act as nonselective inhibitors of both isoforms of COX. It has been proposed that the therapeutic efficacy of anti-inflammatory drugs is primarily the result of COX-2 inhibition. Cyclooxygenase (including COX-2) inhibitors have been developed clinically as a treatment for dysmenorrhea.

Far-infrared radiation offers a nondrug alternative therapy to relieve inflammation by targeting prostaglandin and COX-2. Our earlier study had investigated a FIR-emitting ceramic material with a focus on basic medical science of cells and animal models. We found that the FIR-emitting ceramic material promoted microcirculation and induced other effects by upregulating calcium-dependent nitric oxide (NO) and calmodulin in different cell lines $[5,6]$. We manifested that the FIR ceramic material exerted an antioxidant effect by increasing the hydrogen peroxide-scavenging ability in different cells lines [7, 8]. We also illustrated that FIR induced anti-inflammatory effects by inhibiting prostaglandin (PGE-2) in SW1353 human chondrosarcoma

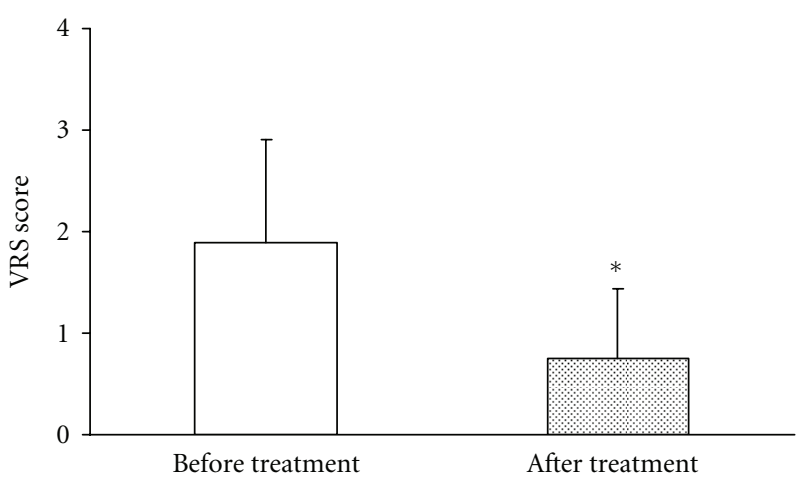

FIGURE 6: The VRS scores before and after treatment. Expression values are the mean and standard deviation, and the statistical difference was detected by Wilcoxon Signed-Rank Test. Asterisk means that this group has statistical difference with control group $(P<0.05)$.

cell line [20]. In addition, our unpublished data show that FIR irradiation had significant inhibition of COX-2 elevation during lipopolysaccharide-induced inflammation in both murine macrophages and human chondrosarcoma cell line.

Ischemia is mainly a consequence of decreased microcirculation or a reduction in local muscle blood flow and perfusion. This scenario has important consequences for cellular metabolic status, with a significantly worsened metabolic and acid-base status while acidosis is accumulated. During dysmenorrhea, the corresponding regional organic ischemia is associated with increased oxidative stress due to increased levels of reactive oxygen species (ROS), such as superoxide and hydrogen peroxide $\left(\mathrm{H}_{2} \mathrm{O}_{2}\right)$, which are responsible of destructive processes in organic tissues $[7,8]$. Our previously published data indicated that FIR-irradiating ceramic material produced calcium-dependent $\mathrm{NO}[5,6]$. It is a medical fact that NO is essential to female-related cells such as uterine and placental cells. During menstruation and pregnancy, women need to produce more NO in the related cells. Recent medical research has indicated that diminished levels of NO induce myometrial contractions, while NO can cause uterine relaxation. By using oral glyceryl trinitrate as a source of NO can relax the exaggerated myometrial contractions in primary dysmenorrhea. A recent review concluded that nitroglycerin significantly reduces the pain in primary dysmenorrheal [21].

Several important merits of NO and the NO-related biomolecules suggest the mechanisms by which dysmenorrhea is alleviated. First, NO inhibits platelet aggregation, reducing inflammation and providing pain relief. Second, adequate NO activity can improve microcirculation, which is a protective factor in maintaining the smooth muscle dilation of blood vessels. Because NO increases blood flow, microcirculation in the endometrial tissue is enhanced, thus relieving inflammation and dysmenorrhea. Third, dysmenorrhea is due to rapid and strong uterine contractions. NO helps coordinate and relieve smooth muscle contraction and relaxation [22]. Fourth, NO is involved in the endocrine mechanism by which menstruation is initiated. Finally, an exogenous NO donor, glyceryl trinitrate, is already in use 


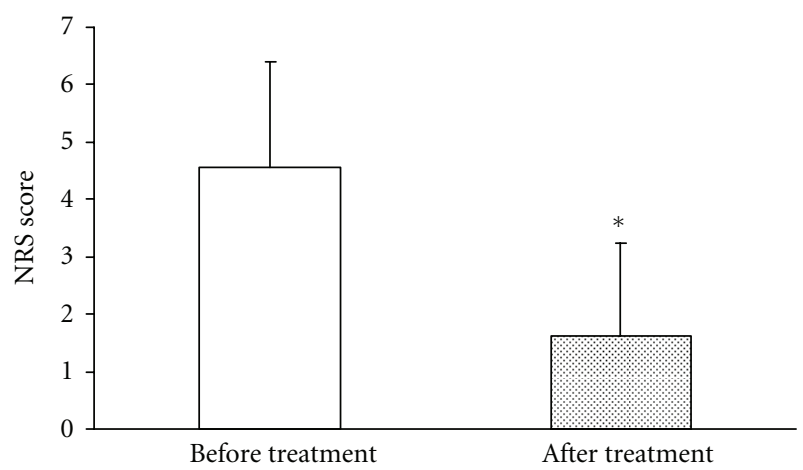

FIgURE 7: The NRS scores before and after treatment. Expression values are the mean and standard deviation, and the difference between groups was analyzed using Wilcoxon Signed-Rank Test. Asterisk means that this group has statistical difference with control group $(P<0.05)$.

as medication to relieve dysmenorrhea by local application. Glyceryl trinitrate has a relaxing effect on the myometrium and has been shown to have a beneficial effect on primary dysmenorrheal [23, 24].

Primary dysmenorrhea as a psychoneurotic disorder is the most common reason for adolescent girls to consult a gynecologist in Taiwan. Primary dysmenorrhea may lead to severe anxiety. If the dysmenorrheal pain can be relieved, anxiety may also be reduced [2]. Some researchers have suggested using cardiac vagal tone as a novel index of stress and stress-induced pain. The role of the parasympathetic nervous system and particularly the vagus nerve in defining stress has been demonstrated [25]. This study finds that the prolonging of the standard deviation of normal-tonormal RR intervals by FIRB has an effect of relaxation. The relaxation response is controlled by the parasympathetic nervous system and particularly the vagus nerve. This relaxation response is a palliative treatment for pain, and the enhancement of parasympathetic tone is important to apply on different chronic pain control. Automatic nervous system pain regulation by parasympathetic activation via the vagal pathways has been shown to exert a beneficial effect on pain and to produce a relaxation response, included in the treatment of dysmenorrhea [25-28].

The thermal effect of the FIRB is also involved in the treatment of primary dysmenorrhea. Thermal effect individually exhibits some degree of relieving symptoms of dysmenorrhea. One of the mechanisms is warm temperature inhibiting sympathetic nerve activities that are responsible of the pain sensation. Besides, the inhibition of sympathetic nerve activity by warm temperature application on lower abdominal or lumbar region might increase uterine blood flow and remove pain-producing substances $[29,30]$. In addition, heat can treat dysmenorrhea through a form of gate control or by altering pain thresholds centrally or through an altered sense of well-being [31].

Although the therapeutic efficacy of the FIRB had been found in this study, there are some limitations in the interpretation of our findings. This is a self-controlled before-and-after experimental study. In spite of the valid physical evidence without a control group, psychological effects may exist in self-report assessments and affect the pain scores. Future studies are suggested to be designed with a larger sample-sized, randomized, and controlled group to further illustrate the psychoneurotic factors.

\section{Conclusions}

This study demonstrated that FIRB is an effective and safe form of therapy for primary dysmenorrhea. Treatment by FIRB resulted in a significant elevation of temperature and abdominal blood flow as well as effective pain relief assayed by the VRS and NRS. Based on these findings and previous literatures, we speculated that the possible effect of FIR on dysmenorrhea may include NO induction, scavenging oxidative stress, and suppression of COX-2 and PGE2 production. FIRB significantly increased the average SDNN value, reflecting that autonomic tone was modulated for relaxation during menstrual pain. This increase in heart rate variability may explain the effect of FIRB on the relationship between pain, anxiety, and activation of the autonomic nervous system. Therefore, it indicates that FIRB may help relieve dysmenorrhea by regulating the autonomic nervous system.

\section{Conflict of Interests}

The authors declare that they have no conflict of interests.

\section{Acknowledgments}

This work was supported by a grant from the National Science Council of Taiwan, R.O.C. (NSC99-2622-E-241-004CC3 and NSC100-2622-E-241-002-CC3). The authors also gratefully acknowledge the help of Roy Sun and Gwo-Diing Lee.

\section{References}

[1] H. M. Chen and C. H. Chen, "Effects of acupressure at the Sanyinjiao point on primary dysmenorrhoea," Journal of Advanced Nursing, vol. 48, no. 4, pp. 380-387, 2004.

[2] C. Alonso and C. L. Coe, "Disruptions of social relationships accentuate the association between emotional distress and menstrual pain in young women," Health Psychology, vol. 20, no. 6, pp. 411-416, 2001.

[3] S. Y. Yu, J. H. Chiu, S. D. Yang, Y. C. Hsu, W. Y. Lui, and $\mathrm{C} . \mathrm{W} . \mathrm{Wu}$, "Biological effect of far-infrared therapy on increasing skin microcirculation in rats," Photodermatology Photoimmunology and Photomedicine, vol. 22, no. 2, pp. 7886, 2006.

[4] S. Inoue and M. Kabaya, "Biological activities caused by farinfrared radiation," International Journal of Biometeorology, vol. 33, no. 3, pp. 145-150, 1989.

[5] T. K. Leung, C. M. Lee, M. Y. Lin et al., "Far infrared ray irradiation induces intracellular generation of nitric oxide in breast cancer cells," Journal of Medical and Biological Engineering, vol. 29, no. 1, pp. 15-18, 2009.

[6] T. K. Leung, Y. S. Lin, Y. C. Chen et al., "Immunomodulatory effects of far-infrared ray irradiation via increasing calmodulin 
and nitric oxide production in raw 264.7 macrophages," Biomedical Engineering-Applications, Basis and Communications, vol. 21, no. 5, pp. 317-323, 2009.

[7] T. K. Leung, Y. S. Lin, C. M. Lee et al., "Direct and indirect effects of ceramic far infrared radiation on the hydrogen peroxide-scavenging capacity and on murine macrophages under oxidative stress," Journal of Medical and Biological Engineering, vol. 31, no. 5, pp. 345-351, 2011.

[8] T. K. Leung, H. F. Shang, D. C. Chen et al., "Effects of far infrared rays on hydrogen peroxide-scavenging capacity," Biomedical Engineering-Applications, Basis and Communications, vol. 23, no. 2, pp. 99-105, 2011.

[9] T. K. Leung, C. F. Chan, P. S. Lai, C. H. Yang, C. Y. Hsu, and Y. S. Lin, "Inhibitory effects of far-infrared irradiation generated by ceramic material on murine melanoma cell growth," International Journal of Photoenergy, vol. 2012, Article ID 646845, 8 pages, 2012.

[10] A. Masuda, Y. Koga, M. Hattanmaru, S. Minagoe, and C. Tei, "The effects of repeated thermal therapy for patients with chronic pain," Psychotherapy and Psychosomatics, vol. 74, no. 5, pp. 288-294, 2005.

[11] K. Matsushita, A. Masuda, and C. Tei, "Efficacy of Waon therapy for fibromyalgia," Internal Medicine, vol. 47, no. 16, pp. 1473-1476, 2008.

[12] C. H. Lee, J. W. Roh, C. Y. Lim, J. H. Hong, J. K. Lee, and E. G. Min, "A multicenter, randomized, double-blind, placebo-controlled trial evaluating the efficacy and safety of a far infrared-emitting sericite belt in patients with primary dysmenorrhea," Complementary Therapies in Medicine, vol. 19, no. 4, pp. 187-193, 2011.

[13] R. M. B. York and I. L. Gordon, "Effect of optically modified polyethylene terephthalate fiber socks on chronic foot pain," BMC Complementary and Alternative Medicine, vol. 9, article 10, 2009.

[14] A. M. Hug, T. Schmidts, J. Kuhlmann, D. Segger, G. Fotopoulos, and J. Heinzerling, "Skin hydration and cooling effect produced by the Voltaren vehicle gel," Skin Research and Technology, vol. 18, pp. 199-206, 2012.

[15] X. Z. West, N. L. Malinin, A. A. Merkulova et al., "Oxidative stress induces angiogenesis by activating TLR2 with novel endogenous ligands," Nature, vol. 467, no. 7318, pp. 972-976, 2010.

[16] K.-M. Chang and C.-W. Shen, "Aromatherapy benefits autonomic nervous system regulation for elementary school faculty in Taiwan," Evidence-based Complementary and Alternative Medicine, vol. 2011, Article ID 946537, 7 pages, 2011.

[17] W. C. Liang, J. Yuan, D. C. Sun, and M. H. Lin, "Changes in physiological parameters induced by indoor simulated driving: effect of lower body exercise at mid-term break," Sensors, vol. 9, no. 9, pp. 6913-6933, 2009.

[18] T. Force, "Heart rate variability: standards of measurement, physiological interpretation and clinical use. Task force of the european society of cardiology and the north american society of pacing and electrophysiology," Circulation, vol. 93, no. 5, pp. 1043-1065, 1996.

[19] A. Fauconnier, E. Dallongeville, C. Huchon, Y. Ville, and B. Falissard, "Measurement of acute pelvic pain intensity in gynecology: a comparison of five methods," Obstetrics and Gynecology, vol. 113, no. 2, pp. 260-269, 2009.

[20] T. K. Leung, C. H. Chen, C. H. Lai et al., "Bone and joint protection ability of ceramic material with biological effects," Chinese Journal of Physiology, vol. 55, no. 1, pp. 47-54, 2012.
[21] M. Y. Dawood, "Primary dysmenorrhea: advances in pathogenesis and management," Obstetrics and Gynecology, vol. 108, no. 2, pp. 428-441, 2006.

[22] I. T. Cameron and S. Campbell, "Nitric oxide in the endometrium," Human Reproduction Update, vol. 4, no. 5, pp. 565-569, 1998.

[23] S. Ghazizadeh, T. Dadkhah, and M. Modarres, "Local application of glyceril trinitrate ointment for primary dysmenorrhea," International Journal of Gynecology and Obstetrics, vol. 79, no. 1, pp. 43-44, 2002.

[24] C. Lees, S. Campbell, E. Jauniaux et al., "Arrest of preterm labour and prolongation of gestation with glyceryl trinitrate, a nitric oxide donor," The Lancet, vol. 343, no. 8909, pp. 13251326, 1994.

[25] S. W. Porges, "Cardiac vagal tone: a physiological index of stress," Neuroscience and Biobehavioral Reviews, vol. 19, no. 2, pp. 225-233, 1995.

[26] S. D. Schaffer and C. B. Yucha, "Relaxation \& pain management: the relaxation response can play a role in managing chronic and acute pain," The American Journal of Nursing, vol. 104, no. 8, pp. 75-78, 2004.

[27] M. Sakakibara, S. Takeuchi, and J. Hayano, "Effect of relaxation training on cardiac parasympathetic tone," Psychophysiology, vol. 31, no. 3, pp. 223-228, 1994.

[28] T. A. Pellino, D. B. Gordon, Z. K. Engelke et al., "Use of nonpharmacologic interventions for pain and anxiety after total hip and total knee arthroplasty," Orthopaedic Nursing/National Association of Orthopaedic Nurses, vol. 24, no. 3, pp. 182-192, 2005.

[29] Y. Nagashima, H. Oda, M. Igaki et al., "Application of heatand steam-generating sheets to the lumbar or abdominal region affects autonomic nerve activity," Autonomic Neuroscience, vol. 126-127, pp. 68-71, 2006.

[30] T. Hosono, Y. Takashima, Y. Morita et al., "Effects of a heatand steam-generating sheet on relieving symptoms of primary dysmenorrhea in young women," Journal of Obstetrics and Gynaecology Research, vol. 36, no. 4, pp. 818-824, 2010.

[31] M. D. Akin, K. W. Weingand, D. A. Hengehold, M. B. Goodale, R. T. Hinkle, and R. P. Smith, "Continuous low-level topical heat in the treatment of dysmenorrhea," Obstetrics and Gynecology, vol. 97, no. 3, pp. 343-349, 2001. 


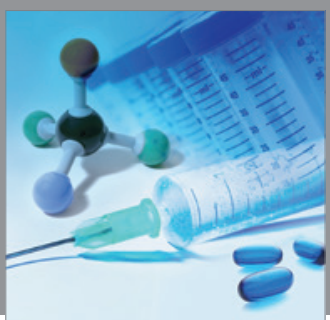

International Journal of

Medicinal Chemistry

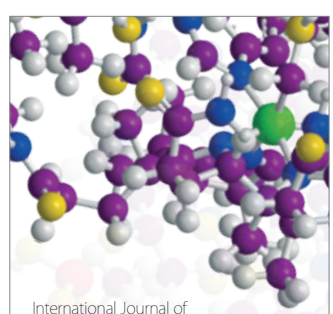

Carbohydrate Chemistry

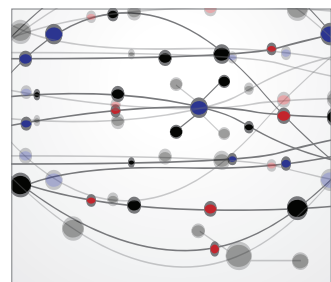

The Scientific World Journal
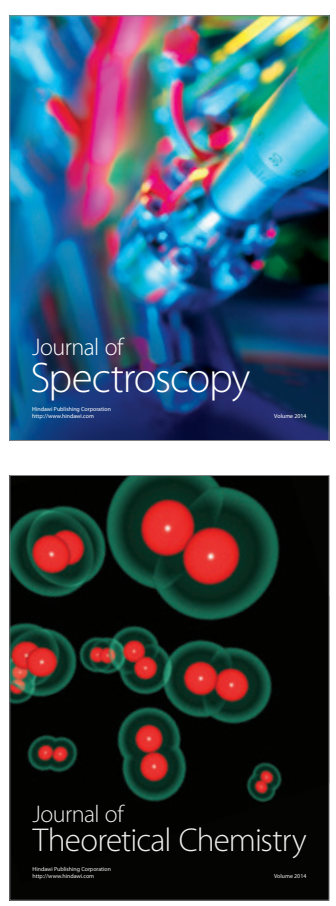
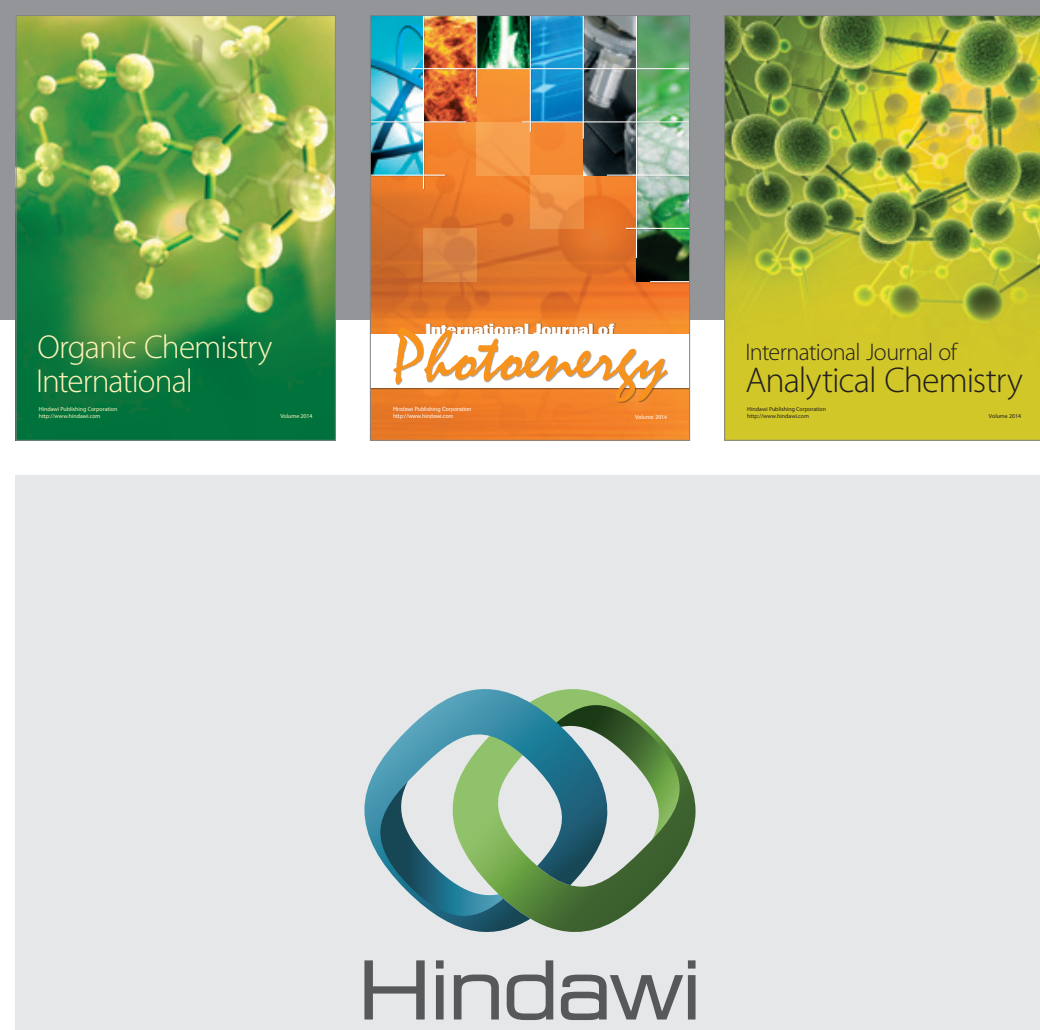

Submit your manuscripts at

http://www.hindawi.com
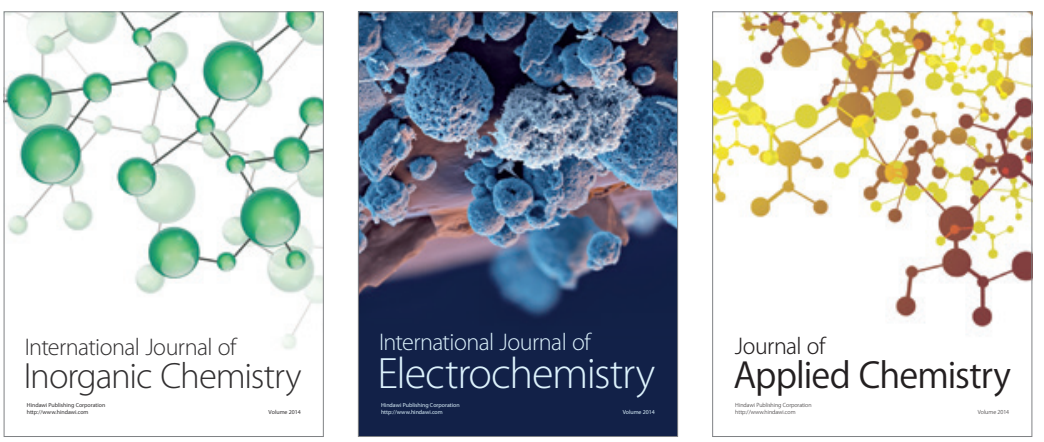

Journal of

Applied Chemistry
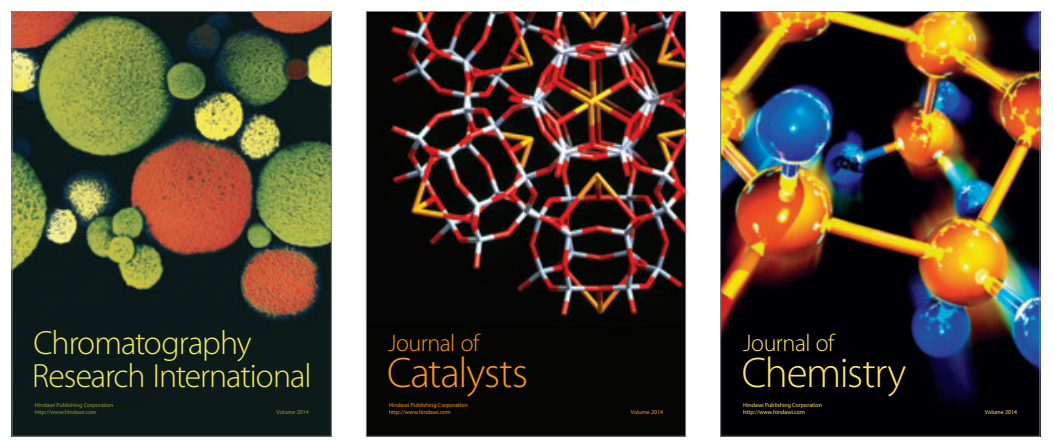
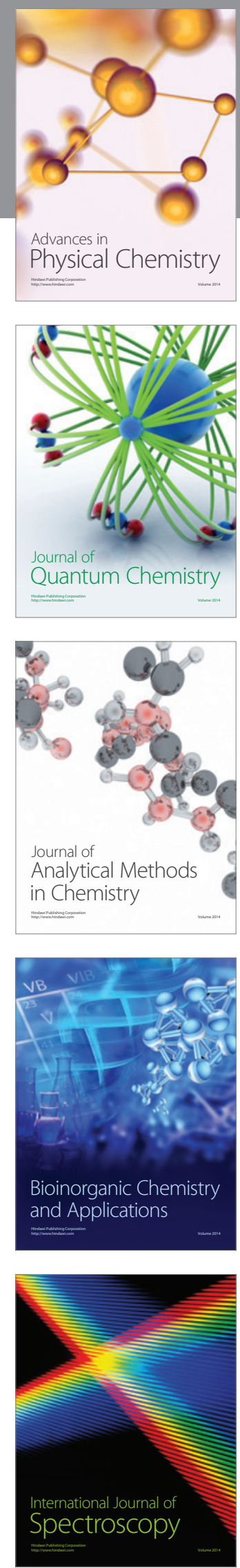